Review

\title{
Non-Invasive Nanoparticle Imaging Technologies for Cosmetic and Skin Care Products
}

\author{
Lynlee L. Lin, Kaitlin L. Nufer, Shoko Tomihara and Tarl W. Prow * \\ Dermatology Research Centre, The University of Queensland, Translational Research Institute, \\ 37 Kent Street, Woolloongabba, Brisbane, Queensland 4102, Australia; E-Mails: 1.li4@uq.edu.au (L.L.L.); \\ k.nufer@uq.edu.au (K.L.N.); s.tomihara@uq.edu.au (S.T.); t.prow@uq.edu.au (T.W.P.) \\ * Author to whom correspondence should be addressed; E-Mail: t.prow@uq.edu.au; \\ Tel.: +61-7-3443-8019; Fax: +61-7-3443-7999.
}

Academic Editor: Lisa A. DeLouise

Received: 26 May 2015 / Accepted: 14 July 2015 / Published: 20 July 2015

\begin{abstract}
The nanotechnology field is growing at an unprecedented rate. This is resulting in significant benefits in skin care products and formulations. Likewise, imaging technology is also advancing. The convergence of these fields offers a unique opportunity to observe and quantify the interactions of nanoparticles within cosmetic and skin care formulations. More importantly, imaging technology holds tremendous promise for understanding how formulated nanoparticles interact with our skin. Imaging technologies can be broken into two major groups that include those that require invasive sample collection and processing (e.g., electron microscopy, mass spectrometry, and super-resolution structured illumination microscopy) and those that can be used in non-invasive data collection settings. Fluorescence microscopy, confocal microscopy, coherent anti-Stokes Raman scattering spectroscopy and optical coherence tomography fall into the latter category and are the focus of this review in the context of skin care product and cosmetics testing. Cosmetic and skin care product testing is most informative when carried out in volunteers. This makes invasive or disruptive analysis techniques unfeasible and supports the use of non-invasive imaging technologies. The combination of non-invasive imaging and minimally invasive microbiopsy sampling for combined imaging and molecular data is the future of skin care product testing.
\end{abstract}

Keywords: non-invasive; imaging; nanoparticles; in vivo; human volunteers; cosmetic testing 


\section{Introduction}

Breakthroughs in material science have led to nano-sized metal particles $(<100 \mathrm{~nm}$ in diameter) to become increasingly available for sunscreen formulations. Nanoparticles are regularly included into skin care products and cosmetics for various reasons. For example, zinc oxide $(\mathrm{ZnO})$ and titanium dioxides $\left(\mathrm{TiO}_{2}\right.$ ) nanoparticles (NPs) are regularly incorporated into sunscreens as physical ultraviolet A (UVA) and ultraviolet $\mathrm{B}$ (UVB) blocking agents. $\mathrm{ZnO}$ and $\mathrm{TiO}_{2}$ at nanoscale level have improved transparency and aesthetic appearance on application [1]. More recently, poly(lactic-co-glycolic acid) (PLGA) nanoparticles as a transdermal delivery carrier were introduced for cosmetic applications [2,3]. As nanotechnology advances, more new nano-materials will be developed. It is imperative to characterise new materials and understand their interactions with complex biological systems such as skin.

The gold standard for nanoparticle imaging and characterisation is electron microscopy. Scanning electron microscopy (SEM) and transmission electron microscopy (TEM) are the two major classes of electron microscopy. SEM is commonly used to evaluate outcomes of cosmetics testing but the technique has also been frequently applied prior to commencing cosmetic studies to predict cosmetic biological interactions upon delivery. Morganti et al. [4] assessed Chitin Nanofibrril-Hyaluronan nanoparticles (CN-HA) for their ability to load an active anti-wrinkle agent and facilitate its penetration across the cutaneous barrier. Characterisation and size of CN-HA nanoparticles by SEM was used to establish the overall release rate of the active ingredient based on the entrapment of the active anti-aging ingredient and the efficacy and degradation profile of the polymer. Matos et al. also employed this technique to determine loading efficiency of minoxidil sulfate to Chitosan nanoparticles [5].

In addition to the characterisation of nanoparticles, SEM was employed to investigate the impact of common hair cosmetic products such as bleaches, dyes and shampoos and conditioners on hair properties [6]. SEM can provide morphological data like changes to hair morphology including breaking or lifting of cuticular scales and cuticle tearing and fragmentation. TEM, on the other hand, is the gold standard for visualising nanoparticles in tissues or cells and their size and distribution [7]. The location of $\mathrm{TiO}_{2}-\mathrm{NP}$ and $\mathrm{ZnO}-\mathrm{NP}$ in sunscreen formulations were identified in vivo in porcine skin by Monteiro-Riviere et al. [8] using both TEM and SEM in UVB treated skin (Figure 1).

Despite the considerable advantages of electron microscopy, there are significant downfalls to this approach as summarised in Table 1. Sample preparation for imaging is a lengthy process and requires a high level of skill and training. Sample collection is invasive and often requires samples to be sectioned, restricting samples to single use only. This process makes real-time assessments and time point studies impractical. Electron microscopy is also prone to generating artefacts from residues from the sample preparation process as well as from nanoparticles themselves. This can make distinguishing nanoparticles from biological materials such as a cellular components or debris challenging [7]. Electron microscopy images also lack contrast in comparison to other non-invasive imaging techniques. Thus, although electron microscopy is a gold standard for materials science, it is not the most appropriate approach for answering questions about the interactions between skin care product formulations and volunteer skin. This review provides a survey of approaches that can be used in the investigation of nanoparticle-skin interactions in the context of cosmetics and skin care products. 
Table 1. Advantages and disadvantages of imaging techniques for nanoparticles associated with cosmetic and skin research.

\begin{tabular}{|c|c|c|}
\hline Technique & Advantages & Disadvantages \\
\hline $\begin{array}{l}\text { Scanning electron microscopy/Transmission } \\
\text { electron microscopy }\end{array}$ & $\begin{array}{l}\text { - High resolution, visualization of individual } \\
\text { nanoparticles. } \\
\text { - Elemental analysis. }\end{array}$ & $\begin{array}{l}\text { - Sample preparation required. } \\
\text { - Requires high level of skill and training. } \\
\text { - Invasive procedure. } \\
\text { - Lack of contrast. }\end{array}$ \\
\hline Single photon laser scanning microscopy & $\begin{array}{l}\text { - Non-invasive procedure. } \\
\text { - Real-time imaging. }\end{array}$ & $\begin{array}{l}\text { - Individual nanoparticles cannot be visualized } \\
\text { due to low resolution. } \\
\text { - Limited penetration depths of up to } 200 \mu \mathrm{m} \text { in } \\
\text { living tissue. } \\
\text { - Requires labelling of samples. }\end{array}$ \\
\hline Multiphoton microscopy & $\begin{array}{l}\text { - Non-invasive procedure. } \\
\text { - Higher penetration depth of up to } 500 \mu \mathrm{m} \text { in } \\
\text { living tissue [9]. } \\
\text { - Real-time imaging. } \\
\text { - Label-free. }\end{array}$ & $\begin{array}{l}\text { - Individual nanoparticles cannot be visualized } \\
\text { due to low resolution. } \\
\text { - Autofluorescence from tissue samples. } \\
\text { - High cost. } \\
\text { - Complexity of system. }\end{array}$ \\
\hline Optical coherence tomography & $\begin{array}{l}\text { - Non-invasive procedure. } \\
\text { - Real-time imaging. } \\
\text { - Label free. } \\
\text { - High penetration depth. } \\
\end{array}$ & $\begin{array}{l}\text { - Individual nanoparticles cannot be visualized } \\
\text { due to low resolution. }\end{array}$ \\
\hline Coherent anti-Stokes Raman Scattering & $\begin{array}{l}\text { - Non-invasive procedure. } \\
\text { - Real-time imaging. } \\
\text { - Label-free. } \\
\text { - Quantitative assessment. }\end{array}$ & $\begin{array}{l}\text { - Individual nanoparticles cannot be visualized } \\
\text { due to low resolution. } \\
\text { - High cost. } \\
\text { - Complexity of system. }\end{array}$ \\
\hline
\end{tabular}




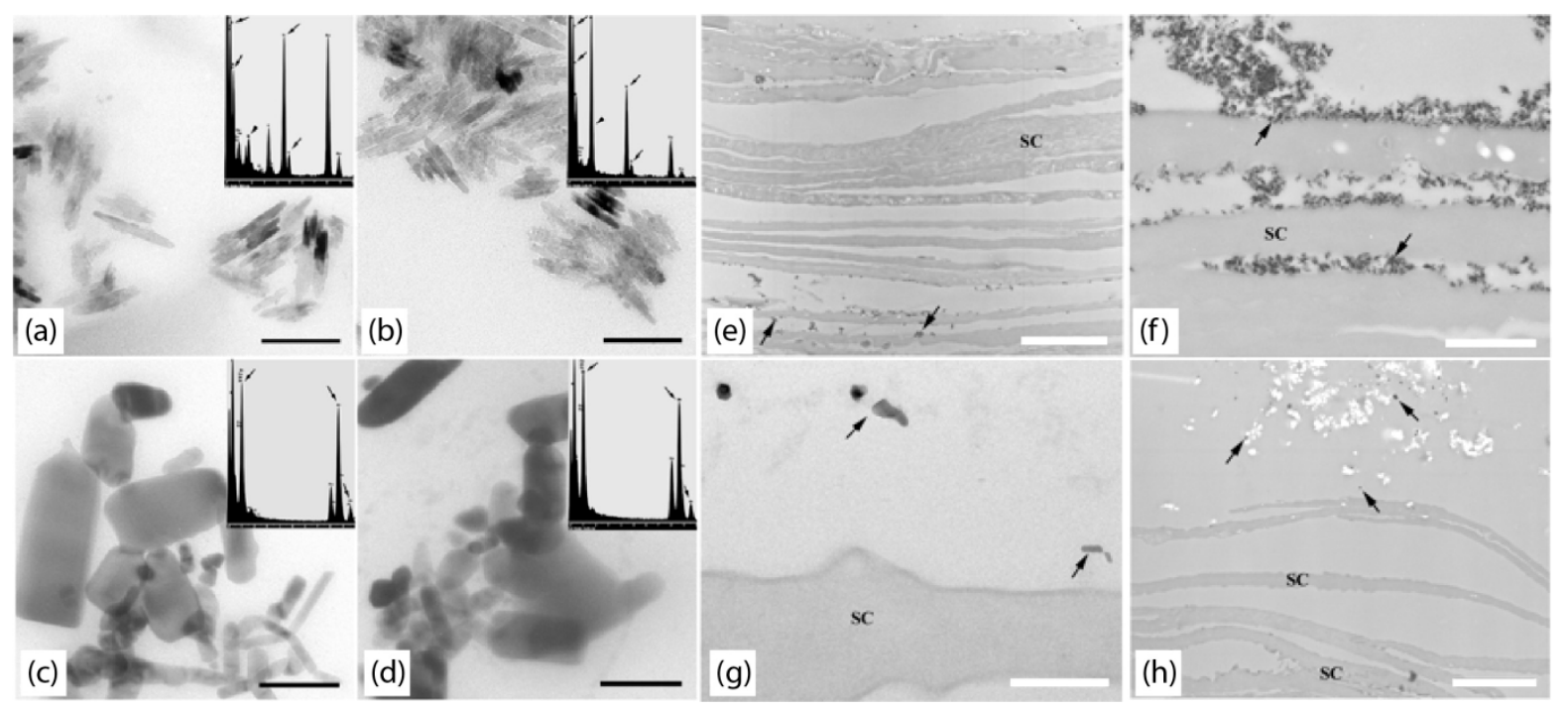

Figure 1. Transmission election micrographs of various diluted $\mathrm{TiO}_{2}$-nanoparticles (NP) in ultrapure water and in porcine skin: (a) CM 630, 10\% $\mathrm{TiO}_{2}$ (T-Lite SF); (b) CM 634, 10\% $\mathrm{TiO}_{2}$ (T-Lite SF); (c) CM 643, 5\% ZnO (Z-COTE HP1); (d) CM 644, 5\% ZnO (Z-COTE). TEM images of stratum corneum are shown of (e) CM 630 treated, UVB-exposed skin; (f) high magnification of CM 634 treated, UVB-exposed skin; (g) CM 643 treated, UVB-exposed skin; (h) CM 644 treated, UVB-exposed skin. The black bars represent $100 \mathrm{~nm}$. The white bars in (e, h), (f) and (g) are $4 \mu \mathrm{m}, 1 \mu \mathrm{m}$ and $250 \mathrm{~nm}$, respectively. Reproduced with permission from [8], published by Oxford University Press, 2011.

\section{Non-Invasive Imaging Techniques}

\subsection{Single-Photon Confocal Microscopy}

Single-photon confocal microscopy, more commonly known as laser scanning confocal microscopy (LSCM) is one of the most widely applied non-invasive, real-time imaging technique. LSCM is a fluorescence-based technique that captures high resolution images by focusing coherent light through a pinhole filter thus removing out of focus light from above or below the specimen before it reaches the detector [10]. The capacity for LSCM to optically section biological sample without compromising its structure provides an option for researchers to investigate and understand nanoparticle interactions with skin, characterise nanoparticle and biomolecule distribution, penetration and overall delivery pathway across the cutaneous barrier. However, the ability of the LSCM to visualise morphological structure of biological samples depends on the set-up of the microscope (i.e., type of laser and optics). The visualisation of cellular morphology of the samples typically requires the use of a laser within visible or near-infrared range.

Reflectance confocal microscopy (RCM) is a form of LSCM that uses the visible to near-infrared spectrum to provide en face optical sections of the skin at a quasi-histological resolution [11]. RCM has been used clinically for the differential diagnosis of basal cell carcinoma [12] and melanoma [13] in addition to a host of other dermatological conditions. We are the first group to assess the penetration profile of topical nanoparticles in human skin using RCM [14,15]. We observed that topically applied nanoparticles were resolved as highly reflective aggregates. In 2011, we investigated the penetration 
profile of gold (Au-NP) and silver (Ag-NP) nanoparticles in ex vivo [14] and in vivo [15] human skin models. One would assume that the potential for nanoparticle penetration would increase if the protective outer barrier of the skin, stratum corneum, is compromised. Tape-stripping protocols were carried out to mimic this type of damage. Both Au-NP and Ag-NP aggregated on the skin surface and within the furrows, and did not enter viable epidermis even when the outer skin barrier was disrupted [14,15]. Our secondary goal in the silver nanoparticle study was also to assess the natural rate of nanoparticle clearance after topical application. Visible aggregates were observed up to 10 days in discrete areas of damaged skin but not in intact skin (Figure 2) [15].

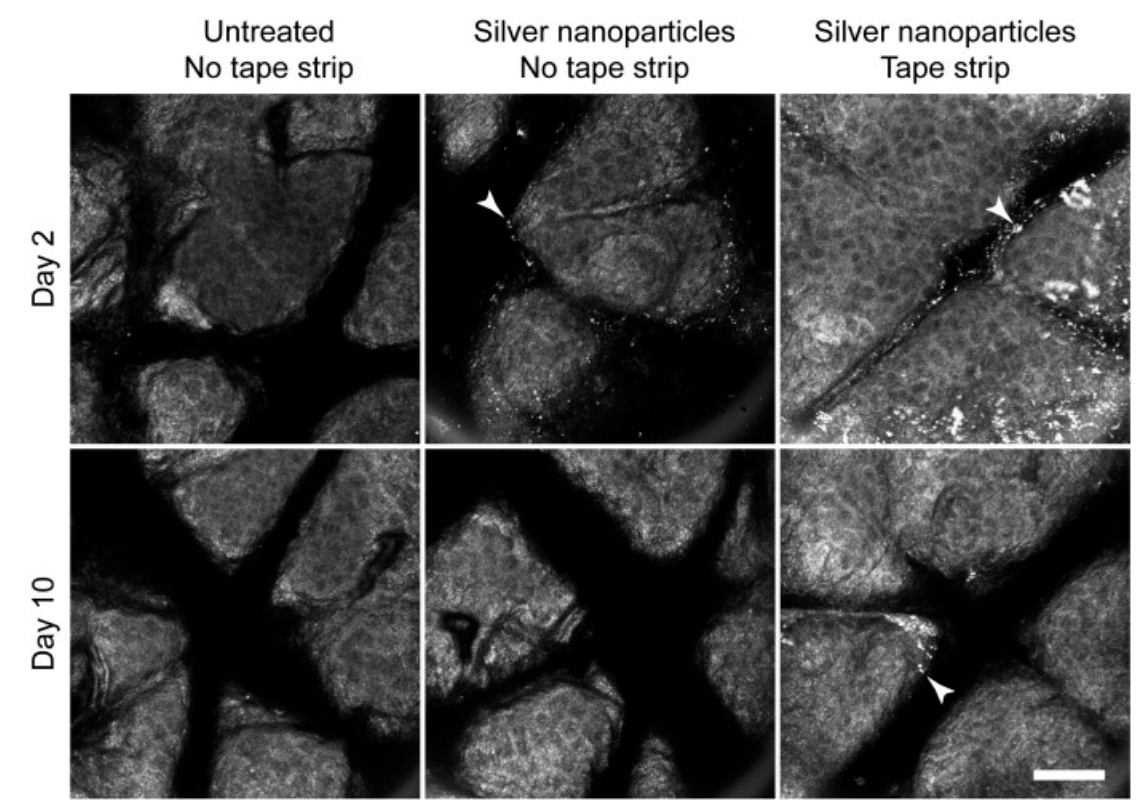

Figure 2. In vivo, en face reflectance confocal sections of human skin applied with ZnO-NP. Silver nanoparticles, indicated by white arrowheads, were seen aggregating on the skin surface for up to 6 days (data not shown) in intact skin and up to 10 days in barrier-disrupted skin. The white bar represents $200 \mu \mathrm{m}$. Reproduced with permission from [15], published by Elsevier B.V., 2011.

Tattoo inks and permanent make-up are also considered by the US Food and Drug Administration (FDA) as cosmetic products. Ink and pigments have been reported to contain carbon nanoparticles [16]. These products are usually administered through injection into the superficial dermis layer but they have not been approved by FDA for such application methods [17]. More than 150 adverse reactions to permanent make-up were reported to the FDA between 2003 and 2004. Maier et al. was the first group to use RCM for investigating the skin alteration in a 40-year-old female's lip after undergoing a permanent lip lining procedure done at a cosmetic institute [18]. The patient reported substantial swelling, scaling and tenderness even after topical treatment with corticosteroid. Pigment as bright clusters of particles dispersed in the epidermis and superficial dermis using high-definition optical coherence tomography (HD-OCT) and RCM. Non-infectious granuloma that was made up of highly reflective structures with macrophages loaded with pigment was detected in both HD-OCT (Figure 3) and RCM images, and was further confirmed by a skin biopsy [18]. 

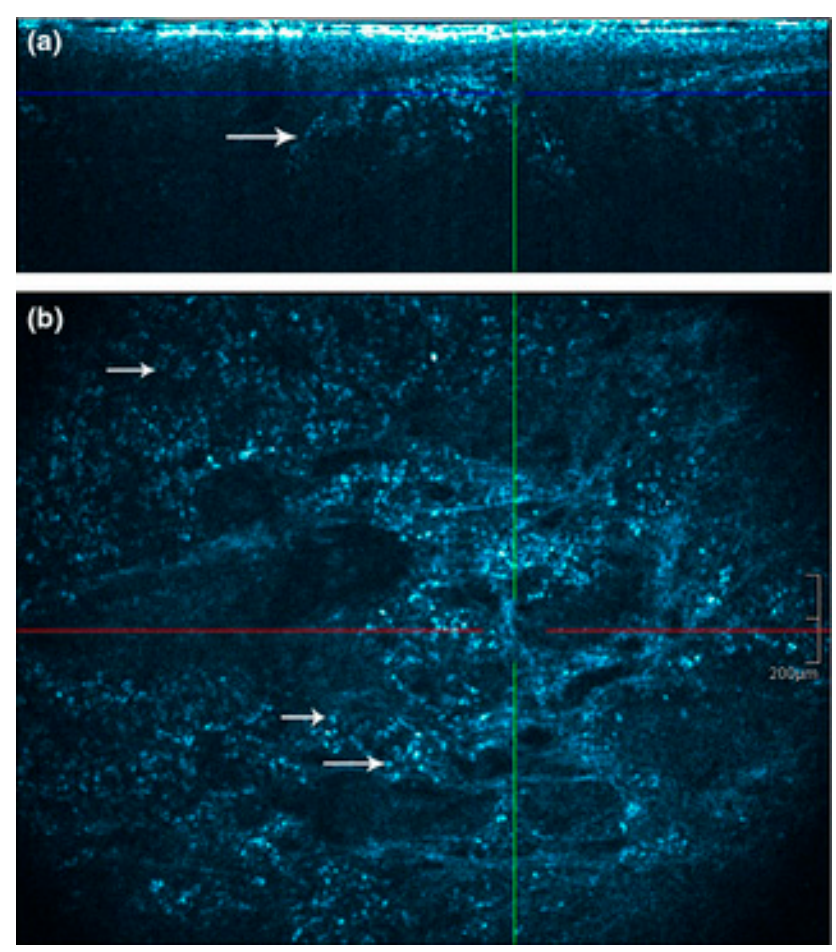

Figure 3. High-definition optical coherence tomography image of lip treated with permanent make-up. Aggregated pigmentation and formation of granuloma-like structures (white arrow) was identified below the epidermis from a cross-sectional image (a). Scattered pigment consisting of bright structures with dark centre that corresponded to pigment loaded macrophage (white arrow) was observed from en face high-definition optical coherence tomography (HD-OCT) image (b). Reproduced with permission from [18], published by European Academy of Dermatology and Venereology, 2014.

Particle size and dispersion are important performance factors for sunscreen products [19]. It is unknown if these properties changes when raw $\mathrm{TiO}_{2}-\mathrm{NP}$ are incorporated into sunscreen formulations. In order to accurately assess these changes, the characterisation methods employed have to be performed without the need to dilute or modify samples. Wokovich and his group employed three different techniques to determine the impact of formulation process to size or shape of $\mathrm{TiO}_{2}-\mathrm{NP}$ [20]. Four different sunscreen formulations were assessed in intact porcine skin: a sunscreen vehicle with no nanoparticles, uncoated $21 \mathrm{~nm} \mathrm{TiO} 2-\mathrm{NP}$, coated $50 \mathrm{~nm} \mathrm{TiO} 2-\mathrm{NP}$ and a submicron sized $250 \mathrm{~nm} \mathrm{TiO} 2-\mathrm{NP}$. LCSM has a resolution limitation of $200 \mathrm{~nm}$ where identification and imaging of individual nanoparticles is not possible. This means that a single nanoparticle signal can theoretically be detected within a single pixel, but the device cannot distinguish whether the signal arises from one or more nanoparticles spatially. Wokovich et al. were able to evaluate nanoparticle dispersion and penetration depth through the skin. LSCM was used in conjunction with invasive methods of higher resolutions including X-ray diffraction (elemental analysis) and SEM, to analyse nanoparticle delivery and penetration to the skin. However, we have found that electron microscopy approaches like this are prone to processing contamination artefacts and low signal strength that make results interpretation difficult. LSCM of sunscreens containing uncoated $\mathrm{TiO}_{2}-\mathrm{NP}$ and submicron $\mathrm{TiO}_{2}-\mathrm{NP}$ saw an even 
distribution and was confirmed by SEM. Coated $\mathrm{TiO}_{2}-\mathrm{NP}$ sunscreen showed uneven particle distribution with SEM confirming particles to appear as aggregates on the skin surface [20].

In addition to the technique's resolution limits, skin and its cellular components are often difficult to analyse using LSCM. Repeated imaging using laser light sources risks irradiation damage in living tissues, particularly for in vivo studies. The generation of autofluorescence while imaging is one of the most problematic obstacles when using LSCM. However, this can be avoided by using a dual channel setup to separate signals from the fluorophore and sample [19]. Laser confocal microscopes also come at a significant purchase and maintenance costs.

\subsection{Multiphoton Microscopy and Time-Correlated Single-Photon Counting}

Multiphoton microscopy (MPM), also known as multiphoton tomography (MPT) offers real-time in vivo imaging and has become a relevant tool for the investigation of nanoparticles in living cells and tissue given its high image resolution, contrast and capacity to make use of skin autofluorescence [21]. MPM utilises femtosecond pulsed lasers to excite endogenous and exogenous fluorophores including unlabelled nanoparticles [7]. MPM is often combined with additional detectors to distinguish signals between nanoparticles and endogenous fluorophores. Time correlated single photon counting (TCSPC) enables fluorescent lifetime imaging (FLIM). The pairing of TCSPC detectors results in a dye free assessment and the ability to quantitate data generated from simultaneous assessments [7].

Multiple studies have been carried out in human volunteers and human skin to determine if $\mathrm{ZnO}$ penetrates beyond the stratum corneum [22-24]. MPM is one of the techniques that were explored by our group to perform in vivo monitoring of $\mathrm{ZnO}-\mathrm{NP}$ on both healthy and diseased skin. Two cohorts of volunteers were included in the study: subjects with no history of skin condition and those who had either psoriasis or atopic dermatitis. ZinClear-S 60 capric/caprylic triglyceride (CCT) nanoparticles, an active ingredient in sunscreens, was applied to the forearm of subjects in doses of 2 and $14 \mathrm{mg} / \mathrm{cm}^{2}$. Volunteers who had healthy, intact skin were subjected to tape-stripping and treated for 4 and $24 \mathrm{~h}$, while those with diseased skin were treated for $2 \mathrm{~h}$. Multiphoton-excited photoluminescence (MEP) signals from $\mathrm{ZnO}-\mathrm{NP}$ was quantified simultaneously with nicotinamide adenine dinucleotide phosphate $(\mathrm{NAD}(\mathrm{P}) \mathrm{H}$, a metabolic intracellular molecule) using multiphoton tomography coupled with TCSPC-FLIM.

MPM images showed $\mathrm{ZnO}-\mathrm{NP}$ signal localised in the stratum corneum and in the furrows of intact and tape-stripped skin at $4 \mathrm{~h}$. Higher levels of ZnO-NP signals were present in the stratum corneum of tape-stripped skin. The ZnO-NP signal was decreased to a minute level in tape-stripped skin after $24 \mathrm{~h}$, suggesting that $\mathrm{ZnO}-\mathrm{NP}$ was dissolved throughout the $24 \mathrm{~h}$ treatment period [23]. Strong $\mathrm{ZnO}-\mathrm{NP}$ signal was detected in the thick stratum corneum layer at 2-h time point. This signal attenuated before the stratum granulosum and was absent in subsequent deeper layers. There was no detectable $\mathrm{ZnO}-\mathrm{NP}$ signal in the superficial viable epidermis both in lesional and non-lesional $\mathrm{ZnO}-\mathrm{NP}$ treated skin [23]. These data support the hypothesis that there is no penetration of $\mathrm{ZnO}-\mathrm{NP}$ into viable human skin when topically applied.

This was further confirmed by another study where we showed that topical nanoparticle signal from sunscreens was removed by washing with soap and water followed by re-imaging (Figure 4) [25]. In our study the $\mathrm{ZnO}-\mathrm{NP}$ were applied topically to the volar forearm of patients, $2 \mathrm{mg} / \mathrm{cm}^{2}$, massaged 
for $30 \mathrm{~s}$ and then exposed for a $2 \mathrm{~h}$ period and then imaged by MPT. Skin was washed with hospital grade soap for $10 \mathrm{~s}$ then re-imaged. The epidermis was analysed by 40 sections of $5.1-\mu \mathrm{m}$ stepwise optical biopsy. ZnO-NP signal intensity was reported as a percentage. Intact skin resulted in a decrease of signal from $36 \% \pm 17 \%$ to $2 \% \pm 1 \%$ after the third wash. Tape-stripped skin resulted in $36 \% \pm 19 \%$ $\mathrm{ZnO}-\mathrm{NP}$ detection before washing, with a decrease to $3 \% \pm 3 \%$ after the third wash. There was no significant difference in $\mathrm{ZnO}-\mathrm{NP}$ signal prior to washing between the intact and tape-stripped skin [25]. Overall this study showed $\mathrm{ZnO}$ penetration evaluated using MPM and that these nanoparticles were able to be localized in the skin using MPT by semi-quantification of signal analysis. ZnO-NP did not penetrate into viable human skin unless the barrier was previously injured. This study also revealed that topical application of $\mathrm{ZnO}-\mathrm{NP}$ can also be removed by washing the site.

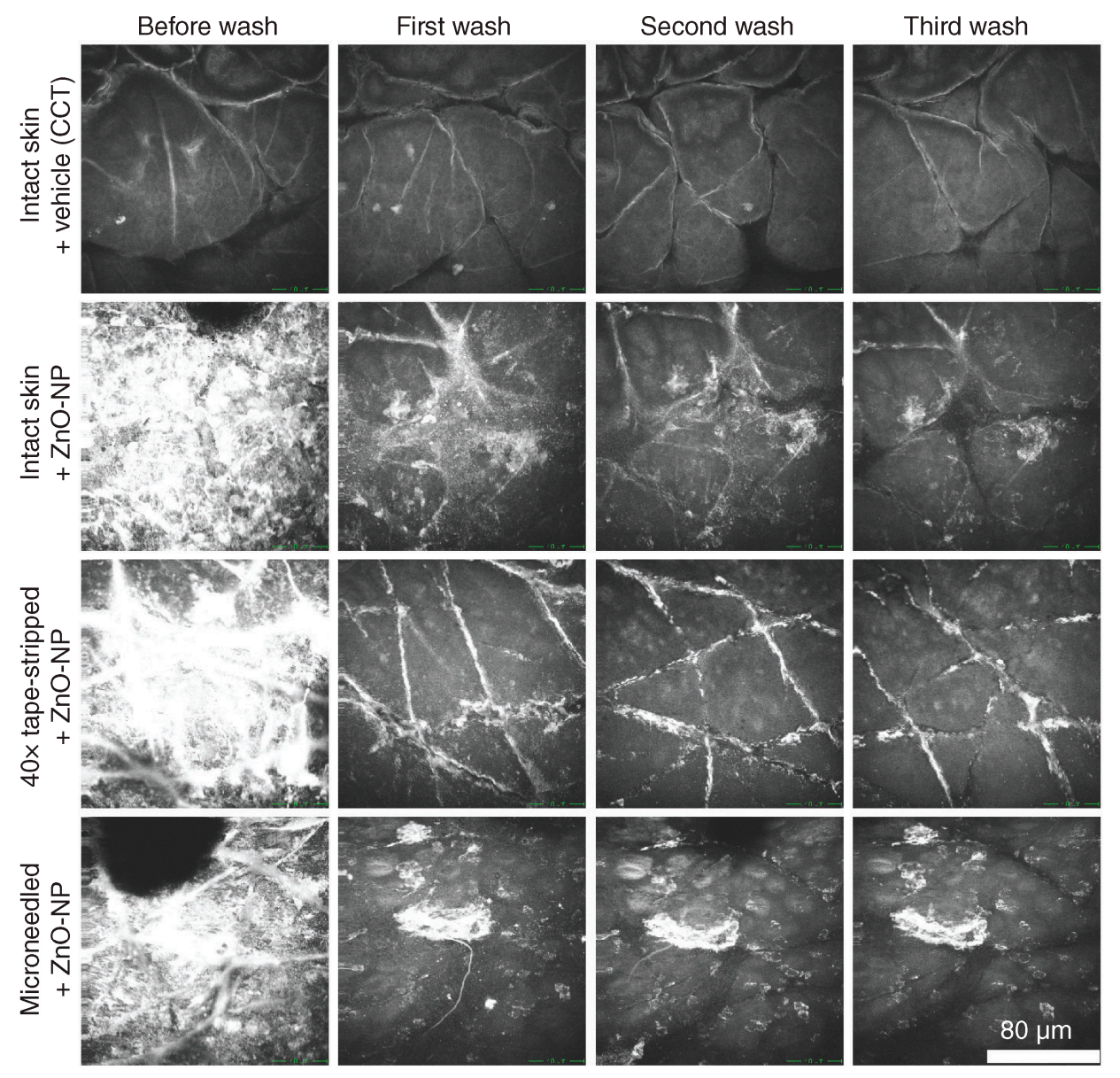

Figure 4. Multiphoton tomography of human skin applied with $\mathrm{ZnO}-\mathrm{NP}$. The rows are representative $z$-projection of the epidermal layers to superficial dermal-epidermal junction before wash, and after subsequent washes. CCT: Capric-caprylic triglyceride buffer. Reproduced with permission from [25], published by Future Medicine Ltd., 2013.

These studies are highly advantageous to cosmetic development and consumer awareness. Due to high purchase, operation and maintenance costs and the requirement for extensive user training, in vivo nanoparticles interactions in human skin are not frequently assessed by multiphoton microscopy. This result was interesting because it showed how easily artefacts from large concentrations of nanoparticles can confound the interpretation of imaging data. 


\subsection{Optical Coherence Tomography}

Optical coherence tomography (OCT) is a non-invasive technique that enables the internal cross-sectional imaging of the microstructural morphology of the skin in real-time. OCT is an emerging clinical imaging technology of inflammatory skin disease, skin cancers and wound healing. Now it is also expanding into the sphere of cosmetic dermatology and cosmetic testing. OCT imaging is done in vivo and captures 2-D or 3-D high resolution images, although these images have lower resolution than confocal techniques. Real-time qualitative and quantitative assessments can then be carried out in the process of cosmetic product testing. The non-invasive nature of this imaging technique also allows for treatment sites to be imaged repeatedly, and monitoring progressive changes in the skin following cosmetic application.

OCT imaging measures the echo time delay of reflected light through low coherence interferometry in the near-infrared wavelength [26]. During OCT imaging, low-coherence near-infrared light is targeted towards an identified site on the skin. Light is then backscattered from the internal microstructures. The magnitude and location of backscattered light from these structures generates a high resolution image [27].

Qualitative assessments of cosmetic products are often performed by OCT. Han et al. carried out a study employing OCT for the examination of the penetration and accumulation of four cosmetic creams to human hand skin and their impact on collagen synthesis levels. One cream contained soluble collagen, another claimed to stimulate collagen production. The remaining two tested creams contained no active ingredients. Swept source optical coherence tomography was performed in vivo in male adult volunteers across a time range from 5 to $120 \mathrm{~min}$. Single intensity from OCT images in hand skin showed an increase in tissue depth in the epidermis only for the cream containing soluble collagen. Peaks were seen at 30 and $120 \mathrm{~min}$ [28]. From OCT images, it was also concluded that in the short term (5-15 min), the collagen soluble cream had no impact on tissue. This information was able to be used by researchers to provide consumers with timeframes to achieve best cosmetic outcomes.

Skin surface roughness and topography for a topical wrinkle reduction therapy was evaluated by Vasquez et al., again testing a topical cosmetic for stimulating collagen production. Changes to participant wrinkle profiles were assessed by calculating frequency distribution depths (FDD). FDD showed number of occurrences increasing in the microstructure region $(0-50 \mu \mathrm{m})$ and decreasing in the fine structure region $(50-170 \mu \mathrm{m})$ for areas that were treated with anti-aging product, with a $10 \%$ reduction in wrinkles. Depth distributions indicated a reduction in occurrence of wrinkles deeper than $170 \mu \mathrm{m}[29]$.

Optical coherence tomography offers many advantages (Table 1) to cosmetic investigators, primarily due to the non-invasive nature of this technique which permits repeated imaging. OCT imaging allows quantitative and qualitative images to be collected and assessed in real time. Time point studies can be carried out with ease to detect changes in treated sites. Imaging of non-transparent tissues also becomes a possibility with OCT due to utilising longer wavelengths of light. 


\subsection{Advanced Raman Spectroscopy}

Advanced label-free imaging technologies with exceptional sensitivity and high time resolution for emerging biomedical applications and medicine are reaching clinical research laboratories in recent years. Raman spectroscopy has been widely used as an analytical and diagnostic tool in various research fields including chemistry [30], physics, material science [31,32] and medicine [33,34] since 1930s. This technique has recently been explored extensively for biomedical applications [35] given its label-free optical imaging ability and the capacity to provide native chemical fingerprints of cells, tissues or biofluids based on inelastic scattering of a light. This inelastic scattering of a photon from a particular molecule is known as the Raman effect. The principle behind molecular chemical fingerprinting is based on the unique vibrational signature of a molecule associated with specific chemical structural bonds. Raman spectroscopy differs from other intrinsic imaging techniques as it is not limited by the number of endogenous fluorophores and is minimally affected by the local environment. A major limitation of this technique is the extremely weak scattering effect due to low photon conversion efficiency of $1: 10^{18}$ resulting in long data acquisition time. Along with the need of high laser power usage and long integration times, the study of living biological specimens is severely limited.

Raman spectroscopy variants of enhanced sensitivity have been developed over the years to overcome current problems of spontaneous Raman scattering. Examples of improved vibrational signals along with video-rate imaging speed Raman spectroscopy is Coherent anti-Stokes Raman Scattering (CARS). A detailed review that covers the principle and set-up of CARS microscopy has been published by Evan and Xie in 2008 [36]. The scope of this review section is to discuss the use of advanced Raman spectroscopy technologies for sunscreen nanoparticles imaging.

Current imaging modalities must overcome existing constraints of localising metal nanoparticles within a structurally complex biological system such as skin to accurately assess their biological interactions. Most optical imaging techniques either lack adequate spatial resolution and/or depth penetration that are required to locate the particles at cellular level within intact tissues. One major limiting factor of existing label-free, non-invasive imaging techniques is the lack of capacity to segregate the metal nanoparticles from the biological specimen without the need of invasive pre-processing. This is with exception to $\mathrm{ZnO}-\mathrm{NP}$, which have intrinsic multi-photon fluorescence properties $[23,37,38]$ as discussed in the earlier sections. CARS microscopy has received considerable attention over the last decade, particularly for biomedical applications. To date, there are no reported data on the use of this technique for assessing sunscreen associated nanoparticles in human volunteers. The use of CARS microscopy for ex vivo detection of $\mathrm{ZnO}-\mathrm{NP}$ and $\mathrm{TiO}_{2}-\mathrm{NP}$ up till now was limited to fish models $[39,40]$.

Moger et al. conducted an exploratory study using this non-invasive technique to locate metal oxide nanoparticles including $\mathrm{ZnO}-\mathrm{NP}$, cerium dioxide $\left(\mathrm{CeO}_{2}\right)$ and $\mathrm{TiO}_{2}-\mathrm{NP}$ in gills of rainbow trout, Onchrhynchus mykiss [39]. They were targeting leaf-like structures, known as the lamella, within the gills of the fish. The structure of the lamella was first identified by comparing the CARS image to a histologically-stained section of the same region (Figure 5a,b). The contrast derived from CARS microscopy that correlated to the high stain uptake areas were carbon-hydrogen $(\mathrm{CH})$ rich structures. Different sizes of the nanoparticles suspended in agarose can be seen from the CARS images, and they 
only observed larger particle aggregations when Forward-CARS (F-CARS) detection scheme was used. Smaller particles that were not present in the F-CARS images were explained either by the reduction in forward-detected signal due to particle-agarose interface or the signal falling below background level. Three-dimensional images of the fish gills that were exposed to $5000 \mu \mathrm{g} / \mathrm{L} \mathrm{TiO}_{2}$ for either $24 \mathrm{~h}$ or 14 days were acquired to determine the location of nanoparticles aggregations within the tissue. They reported small aggregates of particles located in the periphery of lamella after two weeks of exposure (Figure $5 \mathrm{c}-\mathrm{e}$ ). Large clusters of particles of up to $100 \mu \mathrm{m}$ in diameter were also reported to be seen inside the secondary lamella near the capillary after extended period of exposure with CARS microscopy.
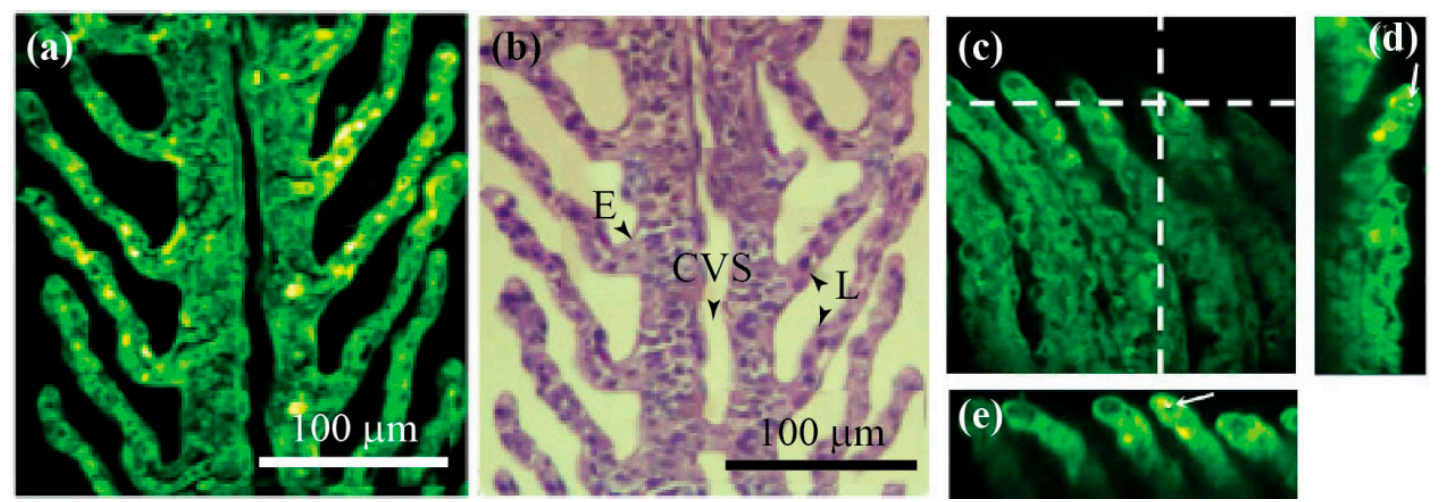

Figure 5. Coherent anti-Stokes Raman Scattering (CARS) image of gill lamella. Tomographic CARS image of $8 \mu \mathrm{m}$ sections, acquired using Stokes and pump wavelengths of $924 \mathrm{~nm}$ and $125 \mathrm{~nm}$, respectively (a). White light image of Schiff-stained section taken at 60× magnification (b), E: Epithelium; CVS: Central venous space; L: Lamella. Example of a trans-axial section of gill lamella that was exposed to $\mathrm{TiO}_{2}$ for two weeks (c). Panels (d) and (e) show cross-sectional image of vertical and horizontal dotted line, respectively [39]. The locations of nanoparticle clusters were highlight by the white arrows in panels (d) and (e).

The group conducted another extensive study in 2010 to investigate the of bioavailability well-characterised metal nanoparticles in zebra fish and rainbow trout [40]. Morphological characterisation of $\mathrm{ZnO}-\mathrm{NP}, \mathrm{CeO}_{2}$ and $\mathrm{TiO}_{2}-\mathrm{NP}$ was confirmed using TEM. Additionally, environmental scanning electron microscopy coupled with energy-dispersive X-ray analysis (ESEM-EDX) was used to determine the spectrum profiles of the nanoparticles in water and tissue samples. Formation of large aggregates was seen in water that was exposed to the nanoparticles on ESEM micrographs while EDX picked up traces of titanium in the samples. There is a high tendency of unmodified nanoparticles, in the absence of natural organic macromolecules, to flocculate into several micrometers in diameter aggregates and precipitate out of solution. This is supported by the lack of significant uptake of zinc in fish tissues. However, they could not confirm if this is lack of uptake is true or due to the masking by high level of $\mathrm{Zn}$ background in the tissues. They have also confirmed for the first time that $\mathrm{TiO}_{2}$-NP enters into the peripheral lamella of the gill through the water column after 14 days of exposure based on CARS analysis [40]. 


\section{Conclusions}

The gold standard for imaging nanoparticles is electron microscopy. This approach works for in vitro imaging but is not appropriate in the complex mixtures or biological environments that are relevant for cosmetics and skin care product testing. Single photon confocal microscopy has evolved into basic science and clinical spaces. Both are useful for examining nanoparticles employed in cosmetics and skin care products. Applications utilising single photon approaches are reliant on fluorescent dyes or the reflective nature of the nanoparticles of interest. The inherent strength of this approach is that it is non-invasive and has the potential to simultaneously collect morphological image data from reflectance imaging. Multiphoton microscopy is conceptually similar to single photon imaging but has some additional benefits and drawbacks. This approach can detect a number of metal nanoparticles with high resolution without the need for fluorescent dyes. Likewise there are a number of autofluorescent molecules in living cells that can be interrogated for information on the metabolic state of nanoparticle treated cells or tissue. It is clear that CARS imaging has great potential for signal separation and quantification in the context of nanoparticles in cosmetics and skin care products, but this has yet to be fully tapped. It seems that the field has significant promise for uptake of imaging technology. The field is also moving quickly in terms of molecular interactions between cosmetic/skin care product nanoparticles and skin responses in complex scenarios. Thus the future of cosmetic and skin care product testing is likely to evolve into more sophisticated imaging followed by minimally invasive skin sampling via microbiopsy for molecular correlation with the imaging outcomes [41-44]. Molecular imaging has the potential to add more substance to claim support and safety assurances.

\section{Acknowledgments}

We thank NHMRC APP1065802 and Epiderm Foundation for the financial support.

\section{Author Contributions}

Lynlee L. Lin contributed to Introduction, Section 2.4, and Conclusion, and edited the manuscript; Kaitlin L. Nufer contributed to Introduction, Sections 2.2 and 2.3; Shoko Tomihara contributed to Section 2.1; Tarl W. Prow edited the manuscript.

\section{Conflicts of Interest}

The authors declare no conflict of interest.

\section{References}

1. Wang, S.Q.; Tooley, I.R. Photoprotection in the era of nanotechnology. Semin. Cutan. Med. Surg. 2011, 30, 210-213.

2. Badihi, A.; Benita, S.; Karra, N.; Nasser, T. Nanoparticles for Cosmetic Applications. Patent WO2012101638 A2, 2 August 2012. 
3. Tsujimoto, H.; Hara, K.; Tsukada, Y.; Huang, C.; Kawashima, Y.; Arakaki, M.; Okayasu, H.; Mimura, H.; Miwa, N. Evaluation of the permeability of hair growing ingredient encapsulated PLGA nanospheres to hair follicles and their hair growing effects. Bioorgan. Med. Chem. Lett. 2007, 17, 4771-4777.

4. Morganti, P.; Palombo, M.; Tishchenko, G.; Yudin, V.; Guarneri, F.; Cardillo, M.; del Ciotto, P.; Carezzi, F.; Morganti, G.; Fabrizi, G. Chitin-hyaluronan nanoparticles: A multifunctional carrier to deliver anti-aging active ingredients through the skin. Cosmetics 2014, 1, 140-158.

5. Matos, B.N.; Reis, T.A.; Gratieri, T.; Gelfuso, G.M. Chitosan nanoparticles for targeting and sustaining minoxidil sulphate delivery to hair follicles. Int. J. Biol. Macromol. 2015, 75, 225-229.

6. Ali, N.; Zohra, R.R.; Qader, S.A.U.; Mumtaz, M. Scanning electron microscopy analysis of hair index on Karachi's population for social and professional appearance enhancement. Int. J. Cosmet. Sci. 2015, 37, 312-320.

7. Prow, T.W. Multiphoton microscopy applications in nanodermatology. Wiley Interdiscip. Rev. Nanomed. Nanobiotechnol. 2012, 4, 680-690.

8. Monteiro-Riviere, N.A.; Wiench, K.; Landsiedel, R.; Schulte, S.; Inman, A.O.; Riviere, J.E. Safety evaluation of sunscreen formulations containing titanium dioxide and zinc oxide nanoparticles in UVB sunburned skin: An in vitro and in vivo study. Toxicol. Sci. 2011, 2011, doi:10.1093/toxsci/kfr148.

9. Sarder, P.; Yazdanfar, S.; Akers, W.J.; Tang, R.; Sudlow, G.P.; Egbulefu, C.; Achilefu, S. All-near-infrared multiphoton microscopy interrogates intact tissues at deeper imaging depths than conventional single- and two-photon near-infrared excitation microscopes. J. Biomed. Opt. 2013, 18, 106012.

10. Claxton, N.S.; Fellers, T.J.; Davidson, M.W. Laser Scanning Confocal Microscopy. Available online: http://www.olympusconfocal.com/theory/LSCMIntro.pdf (accessed on 24 May 2015).

11. Ulrich, M.; Lange-Asschenfeldt, S.; Gonzalez, S. Clinical applicability of in vivo reflectance confocal microscopy in dermatology. G. Ital. Dermatol. Venereol. 2012, 147, 171-178.

12. Nori, S.; Rius-Díaz, F.; Cuevas, J.; Goldgeier, M.; Jaen, P.; Torres, A.; González, S. Sensitivity and specificity of reflectance-mode confocal microscopy for in vivo diagnosis of basal cell carcinoma: A multicenter study. J. Am. Acad. Dermatol. 2004, 51, 923-930.

13. Pellacani, G.; Guitera, P.; Longo, C.; Avramidis, M.; Seidenari, S.; Menzies, S. The impact of in vivo reflectance confocal microscopy for the diagnostic accuracy of melanoma and equivocal melanocytic lesions. J. Investig. Dermatol. 2007, 127, 2759-2765.

14. Labouta, H.I.; Liu, D.C.; Lin, L.L.; Butler, M.K.; Grice, J.E.; Raphael, A.P.; Kraus, T.; El-Khordagui, L.K.; Soyer, H.P.; Roberts, M.S. Gold nanoparticle penetration and reduced metabolism in human skin by toluene. Pharm. Res. 2011, 28, 2931-2944.

15. Prow, T.W.; Grice, J.E.; Lin, L.L.; Faye, R.; Butler, M.; Becker, W.; Wurm, E.M.; Yoong, C.; Robertson, T.A.; Soyer, H.P. Nanoparticles and microparticles for skin drug delivery. Adv. Drug Deliv. Rev. 2011, 63, 470-491.

16. Høgsberg, T.; Löschner, K.; Löf, D.; Serup, J. Tattoo inks in general usage contain nanoparticles. Br. J. Dermatol. 2011, 165, 1210-1218.

17. Tattoos \& Permanent Makeup: Fact Sheet. Available online: http://www.fda.gov/Cosmetics/ ProductsIngredients/Products/ucm108530.htm (accessed on 18 May 2015). 
18. Maier, T.; Flaig, M.; Ruzicka, T.; Berking, C.; Pavicic, T. High-definition optical coherence tomography and reflectance confocal microscopy in the in vivo visualization of a reaction to permanent make-up. J. Eur. Acad. Dermatol. Venereol. 2014, 29, 602-606.

19. Powers, K.W.; Brown, S.C.; Krishna, V.B.; Wasdo, S.C.; Moudgil, B.M.; Roberts, S.M. Research strategies for safety evaluation of nanomaterials. Part VI. Characterization of nanoscale particles for toxicological evaluation. Toxicol. Sci. 2006, 90, 296-303.

20. Wokovich, A.; Tyner, K.; Doub, W.; Sadrieh, N.; Buhse, L.F. Particle size determination of sunscreens formulated with various forms of titanium dioxide. Drug Dev. Ind. Pharm. 2009, 35, 1180-1189.

21. Cicchi, R.; Kapsokalyvas, D.; Pavone, F.S. Clinical nonlinear laser imaging of human skin: A review. BioMed Res. Int. 2014, 2014, doi:10.1155/2014/903589.

22. Darvin, M.; Konig, K.; Kellner-Hoefer, M.; Breunig, H.; Werncke, W.; Meinke, M.; Patzelt, A.; Sterry, W.; Lademann, J. Safety assessment by multiphoton fluorescence/second harmonic generation/hyper-Rayleigh scattering tomography of $\mathrm{ZnO}$ nanoparticles used in cosmetic products. Skin Pharmacol. Physiol. 2012, 25, 219-226.

23. Lin, L.L.; Grice, J.E.; Butler, M.K.; Zvyagin, A.V.; Becker, W.; Robertson, T.A.; Soyer, H.P.; Roberts, M.S.; Prow, T.W. Time-correlated single photon counting for simultaneous monitoring of zinc oxide nanoparticles and NAD (P) H in intact and barrier-disrupted volunteer skin. Pharm. Res. 2011, 28, 2920-2930.

24. Zvyagin, A.V.; Zhao, X.; Gierden, A.; Sanchez, W.; Ross, J.A.; Roberts, M.S. Imaging of zinc oxide nanoparticle penetration in human skin in vitro and in vivo. J. Biomed. Opt. 2008, 13, 064031-064031.

25. Raphael, A.P.; Sundh, D.; Grice, J.E.; Roberts, M.S.; Soyer, H.P.; Prow, T.W. Zinc oxide nanoparticle removal from wounded human skin. Nanomedicine 2013, 8, 1751-1761.

26. Gambichler, T.; Jaedicke, V.; Terras, S. Optical coherence tomography in dermatology: Technical and clinical aspects. Arch. Dermatol. Res. 2011, 303, 457-473.

27. Arevalo, J.F.; Mendoza, A.J.; Fernandez, C.F.; Sanchez, J.G.; Reinaldo, A. Clinical applications of optical coherence tomography in macular diseases. In Retinal Angiography and Optical Coherence Tomography; Springer: New York, NY, USA, 2009; pp. 223-238.

28. Han, S.H.; Yoon, C.H.; Conroy, L.; Vitkin, I.A. OCT monitoring of cosmetic creams in human skin in vivo. Proc. SPIE 2012, 8207, doi:10.1117/12.909504.

29. Vasquez-Pinto, L.; Maldonado, E.; Raele, M.; Amaral, M.; Freitas, A. Optical coherence tomography applied to tests of skin care products in humans-A case study. Skin Res. Technol. 2015, 21, 90-93.

30. Skoulika, S.G.; Georgiou, C.A.; Polissiou, M.G. FT-Raman spectroscopy-Analytical tool for routine analysis of diazinon pesticide formulations. Talanta 2000, 51, 599-604.

31. Mulvaney, P. Surface plasmon spectroscopy of nanosized metal particles. Langmuir 1996, 12, 788-800.

32. Stankovich, S.; Dikin, D.A.; Piner, R.D.; Kohlhaas, K.A.; Kleinhammes, A.; Jia, Y.; Wu, Y.; Nguyen, S.T.; Ruoff, R.S. Synthesis of graphene-based nanosheets via chemical reduction of exfoliated graphite oxide. Carbon 2007, 45, 1558-1565. 
33. Stone, N.; Kendall, C.; Smith, J.; Crow, P.; Barr, H. Raman spectroscopy for identification of epithelial cancers. Faraday Discuss. 2004, 126, 141-157.

34. Shetty, G.; Kendall, C.; Shepherd, N.; Stone, N.; Barr, H. Raman spectroscopy: Elucidation of biochemical changes in carcinogenesis of oesophagus. Br. J. Cancer 2006, 94, 1460-1464.

35. Cao, Y.C.; Jin, R.; Mirkin, C.A. Nanoparticles with Raman spectroscopic fingerprints for DNA and RNA detection. Science 2002, 297, 1536-1540.

36. Evans, C.L.; Xie, X.S. Coherent anti-Stokes Raman scattering microscopy: Chemical imaging for biology and medicine. Annu. Rev. Anal. Chem. 2008, 1, 883-909.

37. Hong, H.; Shi, J.; Yang, Y.; Zhang, Y.; Engle, J.W.; Nickles, R.J.; Wang, X.; Cai, W. Cancer-targeted optical imaging with fluorescent zinc oxide nanowires. Nano Lett. 2011, 11, 3744-3750.

38. Xu, P.; Wen, X.; Zheng, Z.; Kable, E.; Cox, G.; Zhu, H. Two-photon optical characteristics of zinc oxide in bulk, low-dimensional and nano-forms. Proc. SPIE 2005, 5931, doi:10.1117/12.613100.

39. Moger, J.; Johnston, B.D.; Tyler, C.R. Imaging metal oxide nanoparticles in biological structures with CARS microscopy. Opt. Express 2008, 16, 3408-3419.

40. Johnston, B.D.; Scown, T.M.; Moger, J.; Cumberland, S.A.; Baalousha, M.; Linge, K.; van Aerle, R.; Jarvis, K.; Lead, J.R.; Tyler, C.R. Bioavailability of nanoscale metal oxides $\mathrm{TiO}_{2}, \mathrm{CeO}_{2}$, and $\mathrm{ZnO}$ to fish. Environ. Sci. Technol. 2010, 44, 1144-1151.

41. Banan, P.; Lin, L.L.; Lambie, D.; Prow, T.; Soyer, H.P. Effects of ex vivo skin microbiopsy on histopathologic diagnosis in melanocytic skin lesions. JAMA Dermatol. 2013, 149, 1107-1109.

42. Lin, L.L.; Prow, T.W.; Raphael, A.P.; Harrold, R.L., III; Primiero, C.A.; Ansaldo, A.B.; Soyer, H.P. Microbiopsy engineered for minimally invasive and suture-free sub-millimetre skin sampling. F1000Res. 2013, 2, doi:10.12688/f1000research.2-120.v2.

43. McClenahan, P.; Lin, L.L.; Tan, J.M.; Flewell-Smith, R.; Schaider, H.; Jagirdar, K.; Atkinson, V.; Lambie, D.; Prow, T.W.; Sturm, R.A.; et al. BRAFV600E mutation status of involuting and stable nevi in dabrafenib therapy with or without trametinib. JAMA Dermatol. 2014, 150, 1079-1082.

44. Tan, J.M.; Lin, L.L.; Lambie, D.; Flewell-Smith, R.; Jagirdar, K.; Schaider, H.; Sturm, R.A.; Prow, T.W.; Soyer, H.P. BRAF wild-type melanoma in situ arising in a BRAF V600E mutant dysplastic nevus. JAMA Dermatol. 2015, 151, 417-421.

(C) 2015 by the authors; licensee MDPI, Basel, Switzerland. This article is an open access article distributed under the terms and conditions of the Creative Commons Attribution license (http://creativecommons.org/licenses/by/4.0/). 